\title{
Photocharging dynamics in colloidal CdS quantum dots visualized by electron spin coherence
}

\author{
(C) D.R. Yakovlev ${ }^{1,2}$, D.H. Feng ${ }^{1,3}$, V.V. Pavlov ${ }^{2}$, A.V. Rodina ${ }^{2}$, E.V. Shornikova ${ }^{1,4}$, \\ J. Mund ${ }^{1}$, M. Bayer ${ }^{1,2}$ \\ ${ }^{1}$ Experimentelle Physik 2, Technische Universität Dortmund, \\ 44227 Dortmund, Germany \\ ${ }^{2}$ loffe Institute, \\ 194021 St. Petersburg, Russia \\ ${ }^{3}$ State Key Laboratory of Precision Spectroscopy, East China Normal University, \\ Shanghai 200062, China \\ ${ }^{4}$ Rzhanov Institute of Semiconductor Physics, Siberian Branch of the Russian Academy of Sciences, \\ 630090 Novosibirsk, Russia \\ E-mail: dmitri.yakovlev@tu-dortmund.de
}

We use a time-resolved technique with three laser pulses (pump, orientation and probe) to study the photocharging dynamics with picosecond resolution on a long timescale ranging from ps to $\mathrm{ms}$ in CdS colloidal quantum dots. The detection is based on measuring the coherent spin dynamics of electrons, allowing us to distinguish the type of carrier in the dot core (electron or hole). We find that although initially negative photocharging happens because of fast hole trapping on surface states, eventually it evolves to positive photocharging due to electron trapping and hole detrapping. The positive photocharging lasts up to hundreds of microseconds at room temperature.

\section{Acknowledgements}

This work has been supported by the Russian Science Foundation (Grant No 14-42-00015). 